\title{
Role of valence states of adsorbates in inelastic electron tunneling spectroscopy: A study of nitric oxide on $\mathrm{Cu}(110)$ and $\mathrm{Cu}(001)$
}

\author{
Akitoshi Shiotari, ${ }^{1,2,{ }^{*}}$ Hiroshi Okuyama, ${ }^{1}$ Shinichiro Hatta, ${ }^{1}$ Tetsuya Aruga, ${ }^{1}$ Maite Alducin, ${ }^{3,4}$ and Thomas Frederiksen ${ }^{3,5}$ \\ ${ }^{1}$ Department of Chemistry, Graduate School of Science, Kyoto University, Kyoto 606-8502, Japan \\ ${ }^{2}$ Department of Advanced Materials Science, The University of Tokyo, Kashiwa 277-8561, Japan \\ ${ }^{3}$ Donostia International Physics Center (DIPC) - UPV/EHU, E-20018 San Sebastián, Spain \\ ${ }^{4}$ Centro de Física de Materiales, CFM/MPC (CSIC-UPV/EHU), Donostia-San Sebastián, Spain \\ ${ }^{5}$ IKERBASQUE, Basque Foundation for Science, E-48013, Bilbao, Spain
}

(Received 12 June 2016; revised manuscript received 10 August 2016; published 29 August 2016)

\begin{abstract}
We studied nitric oxide (NO) molecules on $\mathrm{Cu}(110)$ and $\mathrm{Cu}(001)$ surfaces with low-temperature scanning tunneling microscopy (STM) and density functional theory (DFT). NO monomers on the surfaces are characterized by STM images reflecting $2 \pi^{*}$ resonance states located at the Fermi level. NO is bonded vertically to the twofold short-bridge site on $\mathrm{Cu}(110)$ and to the fourfold hollow site on $\mathrm{Cu}(001)$. When NO molecules form dimers on the surfaces, the valence orbitals are modified due to the covalent bonding. We measured inelastic electron tunneling spectroscopy (IETS) for both NO monomers and dimers on the two surfaces, and detected characteristic structures assigned to frustrated rotation and translation modes by density functional theory simulations. Considering symmetries of valence orbitals and vibrational modes, we explain the intensity of the observed IETS signals in a qualitative manner.
\end{abstract}

DOI: 10.1103/PhysRevB.94.075442

\section{INTRODUCTION}

Chemical identification of individual molecules adsorbed onto surfaces is one of the most significant challenges for nanoscale investigations using scanning probe microscopes (SPM) such as scanning tunneling microscopy (STM) and atomic force microscopy (AFM). Although SPM gives realspace images with atomic resolution, the topographic images alone usually provide insufficient information to characterize the chemical components of the adsorbates. In order to identify chemical species on surfaces at the single-molecule level, several techniques have been reported: bottom-up fabrication of molecular complexes with manipulation [1], vibrational spectroscopy with tip-enhanced Raman scattering [2], and measuring short-range force curves with AFM [3]. Inelastic electron tunneling spectroscopy (IETS) is also a powerful tool for chemical characterization in the tunneling junction [4]. Characteristic signatures in $d^{2} I / d V^{2}$ spectra on metal-insulator-metal systems, originating from the excitation of vibrational modes of molecules in the insulating layer by the tunneling electrons, were first reported in 1966 [5]. Three decades later, Stipe et al. [6,7] measured vibrational IET spectra of individual molecules on metal surfaces with the STM. Subsequently, STM-IETS has been appreciated as a detection method not only for molecular vibrations but also for surface phonon modes [8] and spin flips of magnetic atoms and molecules $[9,10]$.

In contrast to intuitive selection rules of infrared and Raman spectroscopy, a definitive selection rule of IETS has not been established. Nonetheless, recent theoretical studies have demonstrated valid models for elastic and inelastic tunneling processes, and have successfully reproduced experimental STM-IET spectra [11-20]. IETS for CO on metal surfaces have been thoroughly investigated as a typical model of a simple

\footnotetext{
*shiotari@k.u-tokyo.ac.jp
}

molecule-metal substrate system [12,15,21-30]. $\mathrm{O}_{2} / \mathrm{Ag}(110)$ is also an interesting subject of study [15-17] because on-resonant IETS signals, i.e., dips (peaks) in $d^{2} I / d V^{2}$ at positive (negative) sample biases, are detected [31]. Alducin et al. $[16,17]$ reproduced the IET spectrum theoretically and elucidated that elastic components of the tunneling current become predominant if a resonance state of the adsorbate located just at the Fermi level couples conveniently with vibrational modes. According to the theoretical model, the symmetric property of the molecular orbitals near the Fermi level plays an important role in electron-vibration coupling owing to the inelastic and elastic tunneling processes. Very recently, characteristic on-resonant features were also observed in IETS of $\mathrm{H}_{2} \mathrm{O}$ bonded onto a $\mathrm{NaCl}$ ultrathin film on $\mathrm{Au}(111)$ [32]; the IETS intensities were enhanced by bringing the STM tip closer to the adsorbate, and the lineshapes changed from symmetric dips to asymmetric features in $d^{2} I / d V^{2}$. Based on theoretical modeling, these modifications of the IETS were argued to result from the energy shift of the highest occupied molecular orbital states toward the Fermi level $\left(E_{\mathrm{F}}\right)$ [32]. However, an experimental insight into a correlation between vibrational modes and electronic states of adsorbates still remains insufficient; only few IETS studies in combination with scanning tunneling spectroscopy (STS) have been reported hitherto $[33,34]$.

Nitric oxide (NO) molecules on $\mathrm{Cu}$ surfaces have been prominent systems to monitor the local electronic states with STM images and STS measurements [35-37]. For an isolated NO molecule adsorbed onto $\mathrm{Cu}(110)$ at $\sim 15 \mathrm{~K}$, the resonance state of its valence orbital $\left(2 \pi^{*}\right)$ remains at $E_{\mathrm{F}}$, and therefore, the orbital is visible in the STM image at low bias voltage [36]. Moreover, the electronic states can be modified by formation of covalent bonding to yield a dimer [(NO) $)_{2}$ ] on the surface [35]. Therefore $\mathrm{NO}$ on $\mathrm{Cu}$ surfaces seem to be an ideal model system to investigate a correlation between IETS signals and resonance states. 
In this study, we observed isolated $\mathrm{NO}$ monomers on $\mathrm{Cu}(110)$ and $\mathrm{Cu}(001)$, which are vertically bonded onto the short-bridge site and the hollow site, respectively. On $\mathrm{Cu}(110)$ the doubly degenerate $2 \pi^{*}$ orbital of NO is lifted upon adsorption onto the anisotropic substrate. Conversely, on $\mathrm{Cu}(001)$, the $2 \pi^{*}$ orbital remains degenerate and is located at $E_{\mathrm{F}}$. We also observed $\mathrm{NO}$ dimers $\left[(\mathrm{NO})_{2}\right]$ on these surfaces, fabricated by approaching two NO monomers to each other with STM manipulation. STS measurements clarify their resonance states near $E_{\mathrm{F}}$, which characterize the STM images. We measured IETS for both NO monomers and dimers on the two $\mathrm{Cu}$ surfaces. The experimental observations were also studied theoretically by electronic structure calculations for the role of vibrational excitations in the tunneling process. This enabled us to assign the characteristic peaks in IETS to specific adsorbate vibrational modes and to discuss "propensity rules" based on symmetry considerations of the electron-vibration coupling matrix elements.

\section{METHODS}

The STM experiments were carried out in an ultrahighvacuum chamber at $6 \mathrm{~K}$ (USM-1200, Unisoku). An electrochemically etched tungsten tip was used as an STM probe. Single-crystalline $\mathrm{Cu}(110)$ and $\mathrm{Cu}(001)$ surfaces were cleaned by repeated cycles of $\mathrm{Ar}^{+}$sputtering and annealing. The surfaces were exposed to NO gas via a tube doser positioned $\sim 1 \mathrm{~cm}$ from the surface at up to $15 \mathrm{~K}$, through a variable-leak valve. For STS, $d I / d V$ curves were obtained by using a lock-in amplifier with modulation voltage $V_{\text {mod }}^{\text {(rms) }}=1-4 \mathrm{mV}$ at $590 \mathrm{~Hz}$ with the feedback loop open. Unless otherwise stated, each presented STS spectrum is displayed after subtraction of $d I / d V$ obtained over the bare surface at the same tip height (see Appendix for a discussion of the background subtraction). For IETS, $d^{2} I / d V^{2}$ curves were obtained by using a lock-in amplifier with $V_{\text {mod }}^{(\mathrm{rms})}=6-10 \mathrm{mV}$ at $590 \mathrm{~Hz}$ with the feedback loop open. Each IETS spectrum is displayed after subtraction of $d^{2} I / d V^{2}$ obtained over the bare surface at the same set point (see Appendix).

Electronic structure calculations were performed with density functional theory (DFT) and the GGA-PBE exchangecorrelation functional [38] as implemented in the VASP code [39]. The projected-augmented-wave (PAW) method was used to describe the atomic cores [40,41]. One or two $\mathrm{NO}$ molecules were placed on a five-layer $\mathrm{Cu}$ slab exposing either the (110) or the (001) surface, using a lattice constant of $a=3.64 \AA$. Each slab was separated by about $11 \AA$ of vacuum along the surface normal to reduce the interaction between periodic cells. Similarly, the single-adsorbate(s) limit was modeled with $4 \times 5$ surface cells, except for $\mathrm{NO} / \mathrm{Cu}(001)$ for which a $4 \times 4$ cell was used to improve the symmetry of the low-frequency vibrational modes. We used an energy cutoff of $515 \mathrm{eV}$ in the plane-wave basis set, a $4 \times 4 \times 1$ Monkhorst-Pack sampling of the Brillouin zone, and a firstorder Methfessel-Paxton scheme [42] with a smearing of $50 \mathrm{meV}$ for the orbital occupancies. Forces on the molecule(s) and on the two topmost layers were relaxed to $0.02 \mathrm{eV} / \AA$. Vibrational frequencies and modes were computed using finite displacements of $0.02 \AA$ and a stringent electronic convergence criterion for accurate forces (total energies were converged to $\left.10^{-6} \mathrm{eV}\right)$.

Spin-resolved projected density of states (PDOS) were computed using the implementation of Ref. [43] by projecting for each spin-state $s$ the wave functions of the full system $\psi_{n, s, k}^{\mathrm{TOT}}$ onto the wave functions of the free monomer or dimer $\varphi_{m, s}^{\mathrm{MO}}$, i.e.,

$$
\operatorname{PDOS}_{m, s}(\varepsilon)=\sum_{n, \boldsymbol{k}}\left|\left\langle\varphi_{m, s, \boldsymbol{k}}^{\mathrm{MO}} \mid \psi_{n, s, \boldsymbol{k}}^{\mathrm{TOT}}\right\rangle\right|^{2} \delta\left(\varepsilon-\varepsilon_{n, s, \boldsymbol{k}}\right) .
$$

In this expression, $\sum_{n, \boldsymbol{k}} \delta\left(\varepsilon-\varepsilon_{n, s, \boldsymbol{k}}\right)$ is the density of states (DOS) of all $(n, \boldsymbol{k})$ states with spin $s$, where $n$ and $\boldsymbol{k}$ refer to the band index and $\boldsymbol{k}$ points, respectively. The $\delta$ function is numerically approximated by a Gaussian function with smearing $\sigma=0.2 \mathrm{eV}$. To facilitate comparison to experiments, the PDOS shown in the figures below include a summation over spin.

Constant current STM images were simulated with the Tersoff-Hamann approximation as the local density of states (DOS) integrated within an energy window $\left[E_{\mathrm{F}}, E_{\mathrm{F}}+W\right]$ $\left(\left[E_{\mathrm{F}}+W, E_{\mathrm{F}}\right]\right)$ for positive (negative) $W$ values. IETS maps were computed using the methodology developed by Lorente and coworkers, which is based on the many-body extension of the Tersoff-Hamann theory for the STM [11-14]. Here we used the expressions that take into account finite values of the vibrational quanta and voltage in evaluating the DOS $[16,17]$. Specifically, the relative change in conductance $\Delta \sigma / \sigma$ is evaluated as the sum of the inelastic $\Delta \sigma_{\text {ine }}$ and elastic $\Delta \sigma_{\text {ela }}$ components normalized to the conductance $\sigma$, which are given for positive sample bias $(V>0)$ by

$$
\begin{aligned}
\frac{\Delta \sigma_{\text {ine }}}{\sigma}= & \frac{1}{\rho\left(\boldsymbol{r}_{0}, E_{\mathrm{F}}+e V\right)} \sum_{n, \boldsymbol{k}}\left|\sum_{m} \frac{\left\langle\psi_{m, \boldsymbol{k}}|v| \psi_{n, \boldsymbol{k}}\right\rangle \psi_{m, \boldsymbol{k}}(\boldsymbol{r})}{\varepsilon_{n, \boldsymbol{k}}-\varepsilon_{m, \boldsymbol{k}}+i 0^{+}}\right|^{2} \\
& \times\left[1-f\left(\varepsilon_{n, \boldsymbol{k}}\right)\right] \delta\left(E_{\mathrm{F}}+e V-\hbar \omega-\varepsilon_{n, \boldsymbol{k}}\right)
\end{aligned}
$$

and

$$
\begin{aligned}
\frac{\Delta \sigma_{\text {ela }}}{\sigma}= & \frac{-2 \pi^{2}}{\rho\left(\boldsymbol{r}_{0}, E_{\mathrm{F}}+e V\right)} \sum_{n, \boldsymbol{k}} \mid \sum_{m}\left\langle\psi_{m, \boldsymbol{k}}|v| \psi_{n, \boldsymbol{k}}\right\rangle \psi_{m, \boldsymbol{k}}\left(\boldsymbol{r}_{0}\right) \\
& \times\left.\left[1-f\left(\varepsilon_{m, \boldsymbol{k}}\right)\right] \delta\left(\varepsilon_{m, \boldsymbol{k}}-\hbar \omega-\varepsilon_{n, \boldsymbol{k}}\right)\right|^{2} \\
& \times\left[1-f\left(\varepsilon_{n, \boldsymbol{k}}\right)\right] \delta\left(E_{\mathrm{F}}+e V-\hbar \omega-\varepsilon_{n, \boldsymbol{k}}\right)
\end{aligned}
$$

respectively, where $\sigma$ denotes the differential conductance, $\rho\left(\boldsymbol{r}_{0}, E_{\mathrm{F}}+e V\right)$ is the local DOS at the position $\boldsymbol{r}_{0}$ of the tip apex, and $v$ is the local electron-vibration potential for a vibrational mode with energy $\hbar \omega$. Note that within the Tersoff-Hamann approximation the change in conductance is interpreted as the change in the sample DOS at the tip apex caused by the vibration [11-14]. Equations (2) and (3) give positive and negative $d^{2} I / d V^{2}$ signals, respectively, when $V>0$. In practice, the change in the sample DOS and the local DOS $\rho\left(\boldsymbol{r}_{0}, E_{\mathrm{F}}+e V\right)$ are integrated within an energy window $\left[E_{\mathrm{F}}, E_{\mathrm{F}}+W\right]$ in order to avoid the numerical limitation of having a finite number of states. All simulated topographies and IETS maps correspond to constant density contours of $\rho=5 \times 10^{-9} e / \AA^{3}$. 



FIG. 1. STM images of (a) $\mathrm{NO} / \mathrm{Cu}(110)$ and (b) $\mathrm{NO} / \mathrm{Cu}(001)$ acquired in the constant-current mode with a sample bias $V=30 \mathrm{mV}$ and tunneling current $I=0.5$ for (a) and $5 \mathrm{nA}$ for (b). Meshes represent the lattice of surface $\mathrm{Cu}$ atoms and the black dots represent the position of NO. Recorded $d I / d V$ curves of (c) $\mathrm{NO} / \mathrm{Cu}(110)$ and (d) $\mathrm{NO} / \mathrm{Cu}(001)$. The solid and dashed curves in (c) were recorded over the cross and the empty circle, respectively, in (a). The spectra in (c) $[(d)]$ were acquired by lock-in detection with the bias modulation of $V_{\mathrm{mod}}^{(\mathrm{rms})}=4$ [1] $\mathrm{mV}$ with the feedback disabled at the tip height to give $30 \mathrm{mV}$ and 0.5 [5] $\mathrm{nA}$ over the cross in (a) [(b)]. The inset of (d) shows a $d I / d V$ spatial map of the NO monomer on $\mathrm{Cu}(001)$. The map was acquired at $V=30 \mathrm{mV}$ with $V_{\text {mod }}^{(\mathrm{rms})}=12 \mathrm{mV}$ with the feedback disabled to give $30 \mathrm{mV}$ and $5 \mathrm{nA}$ over the cross in (b). The image sizes are $38 \AA \times 22 \AA$ for (a), $30 \AA \times 20 \AA$ for (b), and $13 \AA \times 11 \AA$ for the inset of (d).

\section{RESULTS AND DISCUSSION}

\section{A. STM observations of NO monomers}

Figures 1(a) and 1(b) show typical STM images of $\mathrm{Cu}(110)$ and $\mathrm{Cu}(001)$, respectively, exposed to $\mathrm{NO}$ at $\sim 12 \mathrm{~K}$. Each image was obtained at $6 \mathrm{~K}$. STM images of $\mathrm{NO} / \mathrm{Cu}(110)$ have been reported previously [35]; dumbbell-shaped protrusions with an apparent height of $\sim 0.2 \AA$ are assigned to NO monomers bonded onto the short-bridge sites $\left(C_{2 \mathrm{v}}\right.$ point group). The image shape of the monomer reflects the $2 \pi^{*}$ orbital along the [110] direction $\left(2 \pi_{[1 \overline{1} 0]}^{*}\right)$ of the molecule bonded vertically to the short-bridge site. The red solid curve in Fig. 1(c) shows a $d I / d V$ spectrum recorded over the protrusion [red cross in Fig. 1(a)]. The resonance state of $2 \pi_{[1 \overline{1} 0]}^{*}$ is located at $E_{\mathrm{F}}$ and contributes predominantly to the STM images at low bias voltage. The resonance state of $2 \pi^{*}$ along the [001] direction $\left(2 \pi_{[001]}^{*}\right)$ is located around $E_{\mathrm{F}}+0.5 \mathrm{eV}$ [36]. Therefore the intrinsic double degeneracy of the $2 \pi^{*}$ orbital is lifted upon adsorption onto the anisotropic substrate.

Conversely, STM images of $\mathrm{NO} / \mathrm{Cu}(001)$ reveal a ringshaped protrusion with an apparent height of $\sim 0.1 \AA$ [Fig. 1(b)]. The image is centered at the hollow site, which is determined by coadsorbed $\mathrm{CO}$ molecules bonded on top of $\mathrm{Cu}$ atoms [21] (not shown). Note that this adsorption site is in contradiction with a previous report based on infrared spectroscopy [44]. The $d I / d V$ curve recorded over the protrusion [blue cross in Fig. 1(b)] displays a resonance located at $E_{\mathrm{F}}$ [Fig. 1(d)]. The inset of Fig. 1(d) shows a $d I / d V$ map at $30 \mathrm{mV}$, indicating that the resonance state originates from the isotropic $2 \pi^{*}$ orbital. Therefore the NO monomer is bonded vertically to the hollow site ( $C_{4 \mathrm{v}}$ point group) with a doubly degenerate $2 \pi^{*}$ orbital.

Figures 2(a) and 2(b) show $d^{2} I / d V^{2}$ curves for $\mathrm{NO} / \mathrm{Cu}(110)$ and $\mathrm{NO} / \mathrm{Cu}(001)$ with a characteristic feature around $V= \pm 19$ and $\pm 25 \mathrm{mV}$, respectively. Each spectrum was measured over the center of the image (i.e., on top of the molecule). The structure in $d^{2} I / d V^{2}$, also directly observable in $d I / d V$ over NO [dashed curve in Fig. 1(c)], corresponds to the increase of inelastic tunneling conductance due to vibrational excitation. We note that it cannot simply be ascribed to the derivative of the comparably smooth $2 \pi^{*}$ resonance itself. The width of the vibrational fingerprint is relatively broad (full width at half maximum of $\sim 30 \mathrm{meV}$ ), suggesting that a few vibrational modes contribute to the structure. The assignment of the peaks will be described later.

We also acquired simultaneous topography and spatial maps of the $d^{2} I / d V^{2}$ intensities at the corresponding bias voltages [Figs. 2(c) and 2(d) for $\mathrm{NO} / \mathrm{Cu}(110)$ and 2(e) and 2(f) for $\mathrm{NO} / \mathrm{Cu}(001)]$. Despite different topographic appearances of NO on the two surfaces, the IETS intensity is in both cases centered on the molecule.

\section{B. DFT calculations of NO monomers}

We conducted spin-polarized DFT calculations for both $\mathrm{NO} / \mathrm{Cu}(110)$ and $\mathrm{NO} / \mathrm{Cu}(001)$. The energetic and geometric properties of the systems, listed in Table I, are basically in agreement with very recent DFT studies [45,46]. On $\mathrm{Cu}(110)$, we find that a single $\mathrm{NO}$ molecule preferentially adsorbs on the short-bridge site in an upright configuration. Note, however, that we are unable to explain the relative stabilities of the upright and bent ("flat-lying") structures reported in Ref. [47] (the bent configuration is computed to be metastable). While the intrinsic spin polarization of $\mathrm{NO}$ is generally reported to be lost upon adsorption on $\mathrm{Cu}$ surfaces [48,49], we found magnetic solutions for upright $\mathrm{NO} / \mathrm{Cu}(110)$ using different $k$ meshes, smearing values, and surface coverages, albeit 


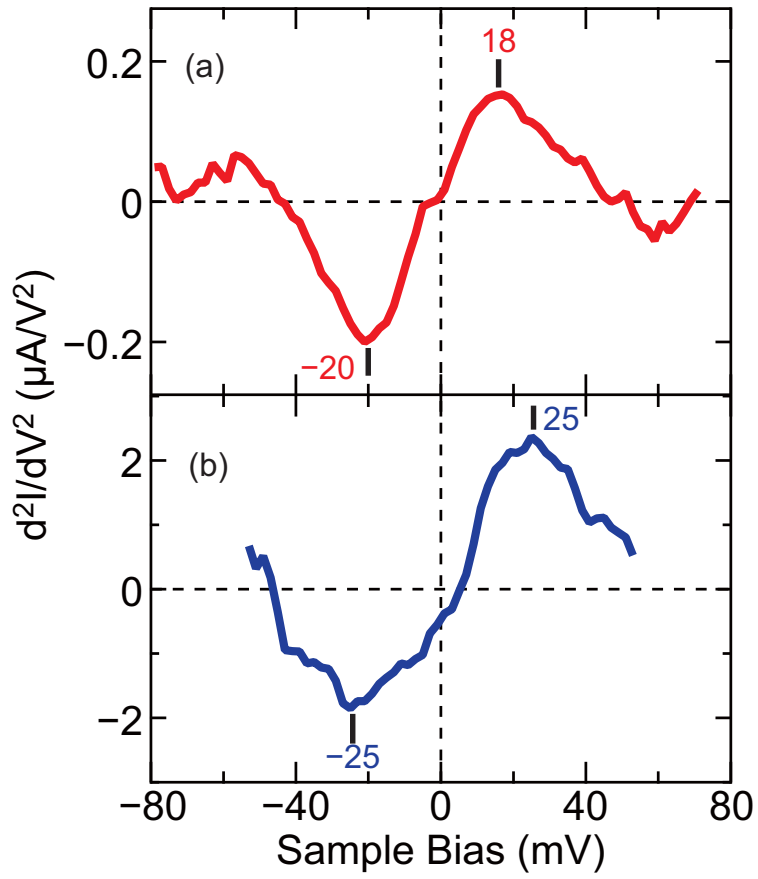

(c) $\mathrm{NO} / \mathrm{Cu}(110)$

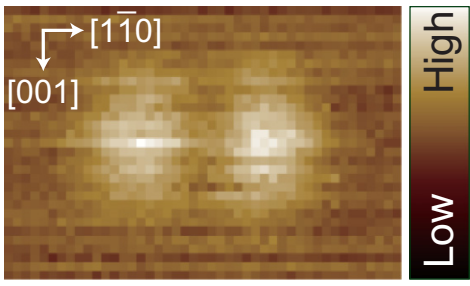

(d)

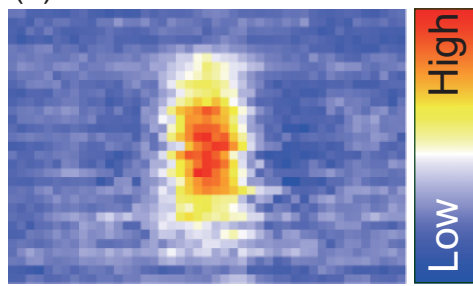

(e) $\mathrm{NO} / \mathrm{Cu}(001)$

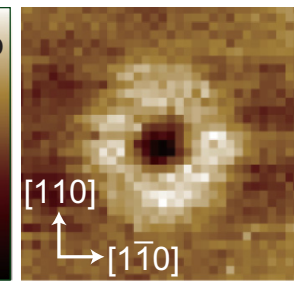

(f)

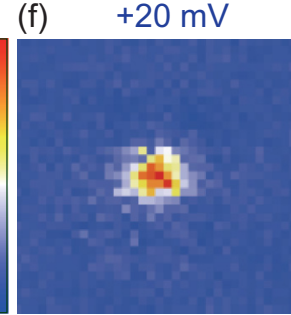

FIG. 2. $d^{2} I / d V^{2}$ curves of (a) $\mathrm{NO} / \mathrm{Cu}(110)$ and (b) $\mathrm{NO} / \mathrm{Cu}(001)$ recorded over on top of the molecule. The spectrum in (a) [(b)] was acquired by lock-in detection with the bias modulation of $V_{\bmod }^{(\mathrm{rms})}=$ 6 [10] $\mathrm{mV}$ with the feedback disabled at the tip height to give $30 \mathrm{mV}$ and 0.5 [5] nA. (c) [(e)] Topographic image and (d) [(f)] $d^{2} I / d V^{2}$ spatial map of the monomer on $\mathrm{Cu}(110)[\mathrm{Cu}(001)]$. The topographic image in (c) [(e)] was acquired with $V=30 \mathrm{mV}$ and $I=0.5$ [5] nA to give the tip height for measuring $d^{2} I / d V^{2}$ signal at each point. The map in (d) [(f)] was subsequently acquired at $V=16$ [20] $\mathrm{mV}$ with $V_{\text {mod }}^{(\mathrm{rms})}=12[10] \mathrm{mV}$ with the feedback disabled. The image sizes are $16 \AA \times 11 \AA$ for (c) and (d) and $11 \AA \times 11 \AA$ for (e) and (f).

the energy difference to the corresponding spin-degenerate calculation was at most a few meV/cell. With the tetrahedron method on a very dense $12 \times 12 \Gamma$-centered $k$ grid the spin polarization eventually disappeared (Table I). The PDOS of $\mathrm{NO} / \mathrm{Cu}(110)$ is shown in Fig. 3(a). The resonance states of $2 \pi_{[110]}^{*}$ and $2 \pi_{[001]}^{*}$ orbitals are nondegenerate and located near $E_{\mathrm{F}}$, compatible with STS data (Fig. 1(c) and Ref. [36]).

Figures 3(b) and 3(c) show simulated topographies of $\mathrm{NO} / \mathrm{Cu}(110)$ for negative and positive $W$, respectively. The former reflects the shape of $2 \pi_{[1 \overline{1} 0]}^{*}$, whereas the latter corresponds to the sum of the $2 \pi_{[1 \overline{1} 0]}^{*}$ and $2 \pi_{[001]}^{*}$ features, in qualitative agreement with STM images acquired at different bias voltages [36]. However, the computed splitting between $2 \pi_{[001]}^{*}$ and $2 \pi_{[1 \overline{1} 0]}^{*}$ is relatively weaker than experimentally observed. More precisely, the maxima of the corresponding PDOS are separated by less than $300 \mathrm{meV}$ and the resonances, which are rather broad, overlap at both sides of $E_{\mathrm{F}}$ within a large energy interval ([ $\left.\left.E_{\mathrm{F}}-1 \mathrm{eV}, E_{\mathrm{F}}+1 \mathrm{eV}\right]\right)$. Consequently, the simulated topography for a sampling of the density of states in the energy interval $\left[E_{\mathrm{F}}, E_{\mathrm{F}}+0.2 \mathrm{eV}\right]$ exhibits a ring-shaped protrusion [Fig. 3(c)], another manifestation of the strongly overlapping resonances above $E_{\mathrm{F}}$ in the DFT electronic structure. However, the experimentally observed $2 \pi_{[1 \overline{1} 0]}^{*}$ orbital can be reasonably reproduced by simulations involving only the occupied states $\left[E_{\mathrm{F}}-0.2 \mathrm{eV}, E_{\mathrm{F}}\right]$, see Fig. 3(b). We note that we explored two mechanisms that could potentially increase the orbital splitting on $\mathrm{Cu}(110)$ in the calculations: (i) NO was forced closer to the substrate by $0.4 \AA$, representing a possible underestimate of the binding distance to the substrate and therefore of the crystal field splitting and (ii) we performed $\mathrm{DFT}+U$ calculations with $U=1-4 \mathrm{eV}$ to favor spin splitting. However, while the quantitative details changed, the relative separation between $2 \pi_{[001]}^{*}$ and $2 \pi_{[110]}^{*}$ was not significantly altered.

Figure 4 shows simulated IETS maps for each of the normal modes for $\mathrm{NO} / \mathrm{Cu}(110)$. The energy window $W=-0.2 \mathrm{eV}$ and constant density contour of $\rho=5 \times 10^{-9} e / \AA^{3}$ were used to maximize features of the experimental $2 \pi_{[110]}^{*}$ resonance. Schematic illustrations of the corresponding vibrations are shown as insets. It is noteworthy that the simulated IETS intensities and shapes strictly depend on the symmetry of the vibrational modes. The maps of the $\mathrm{N}-\mathrm{O}$ stretch (v) and center-of-mass stretch (CM) modes [Figs. 4(a) and 4(c), respectively; $A_{1}$ symmetry] have no intensity, whereas those of the frustrated rotation and translation modes along the [11 10$]$ direction $\left[\mathrm{FR}_{[1 \overline{1} 0]}\right.$ and $\mathrm{FT}_{[1 \overline{1} 0]}$ modes shown in Figs. 4(b) and 4(e), respectively; $B_{2}$ symmetry] have intensities on top of NO. The maps of $\mathrm{FR}_{[001]}$ and $\mathrm{FT}_{[001]}$ modes [Figs. 4(d) and 4(f), respectively; $\mathrm{B}_{1}$ symmetry] also have intensities on top of the molecule but they are comparatively weaker than those of the $\mathrm{B}_{2}$ modes. The calculated vibrational energies (Fig. 4) indicate that the measured $d^{2} I / d V^{2}$ structure around $\pm 19 \mathrm{mV}$ [Fig. 2(a)] should be assigned mainly to the $\mathrm{FT}_{[1 \overline{1} 0]}$ mode $(18 \mathrm{meV})$. The simulated IETS distribution of the FT $_{[1 \overline{1} 0]}$ mode is in good agreement with the measured map [Fig. 2(d)]. The exceptionally broad peak width in Fig. 2(a) further suggests that the observed spectrum could include masked contributions as shoulders from $\mathrm{FR}_{[001]}(20 \mathrm{meV})$ and $\mathrm{FR}_{[1 \overline{1} 0]}(42 \mathrm{meV})$.

The symmetry dependence of the IETS maps can be explained in terms of "propensity rules" proposed by Lorente et al. [11,13,15-17]. Equations (2) and (3) show that an IET signal requires $\left\langle\psi_{m, k}|v| \psi_{n, k}\right\rangle$ to be nonzero, i.e., that an IETS feature is "allowed" if the following equation holds [50]:

$$
\Gamma_{m} \otimes \Gamma_{\mathrm{vib}} \otimes \Gamma_{n}=\Gamma_{\mathrm{sym}},
$$

where $\Gamma_{n(m)}$ denotes the symmetry representation of the $\psi_{n, \boldsymbol{k}(m, \boldsymbol{k})}$ states involved in the electron-vibration coupling induced during the tunneling process, $\Gamma_{\text {vib }}$ denotes the symmetry 
TABLE I. Calculated GGA-PBE adsorption energies, geometric properties (atom-atom distances and angle of the NO axis with respect to the surface plane), and magnetization of $\mathrm{NO}$ molecules on $\mathrm{Cu}(110)$ and $\mathrm{Cu}(001)$.

\begin{tabular}{lcccccc}
\hline \hline System & Site & $\begin{array}{c}E_{\mathrm{ads}}{ }^{\mathrm{a}} \\
(\mathrm{eV})\end{array}$ & $\begin{array}{c}d_{\mathrm{N}-\mathrm{O}} \\
(\AA)\end{array}$ & $\begin{array}{c}\text { Angle } \\
\left({ }^{\circ}\right)\end{array}$ & $\begin{array}{c}d_{\mathrm{N}-\mathrm{N}} \\
(\AA)\end{array}$ & $\begin{array}{c}d_{\mathrm{O}-\mathrm{O}} \\
(\AA)\end{array}$ \\
\hline $\mathrm{NO} / \mathrm{Cu}(110)$ & short-bridge & 1.190 & 1.21 & 0.0 & $0.68(0.00)$ \\
& short-bridge & 0.866 & 1.33 & 78.2 & & $0.00(0.00)$ \\
$\mathrm{NO} / \mathrm{Cu}(001)$ & hollow & 1.257 & 1.24 & 0.1 & $0.00(0.00)$ \\
$(\mathrm{NO})_{2} / \mathrm{Cu}(110)$ & short-bridge & 1.277 & 1.20 & 6.6 & 2.80 & 2.53 \\
$(\mathrm{NO})_{2} / \mathrm{Cu}(001)$ & hollow & 1.226 & 1.23 & 5.4 & 2.88 & 2.65 \\
\hline \hline
\end{tabular}

a The adsorption energy per NO molecule is defined as $E_{\text {ads }}=\left(n \times E_{\mathrm{NO}}+E_{\text {surf }}-E_{\text {system }}\right) / n$, where $E_{\mathrm{NO}}$ is the energy of a single gas-phase $\mathrm{NO}, E_{\text {surf }}$ is the energy of the clean surface, and $E_{\text {system }}$ the energy of the combined system with $n$ NO molecules.

${ }^{b}$ Numbers in parentheses were obtained using the tetrahedron method on a very dense $12 \times 12 \Gamma$-centered $k$ grid.

representation of the corresponding vibrational mode, and $\Gamma_{\text {sym }}$ denotes the totally symmetry representation (e.g., $\mathrm{A}_{1}$ for the $C_{n \text { v }}$ point group).

As an example, Fig. 5 shows simulated maps of the separate inelastic and elastic tunneling components for the $\mathrm{FT}_{[1 \overline{1} 0]}$ mode. The inelastic map shows a positive intensity on top of NO [Fig. 5(a)], which dominates the total IETS map of Fig. 4(e). This can be explained by "propensity rules" [Eq. (4)] as follows. The $\delta$ function in Eq. (2) determines that the $\psi_{n, k}$ state during the tunneling process is the $2 \pi_{[1 \overline{1} 0]}^{*}$ state [Figs. 1(c) and 3(a)], i.e., $\Gamma_{n}=\mathrm{B}_{2}$. The $\mathrm{FT}_{[1 \overline{1} 0]}$ mode gives $\Gamma_{\mathrm{vib}}=\mathrm{B}_{2}$. Thus, in order to satisfy Eq. (4), $\Gamma_{m}$ is required to be $\mathrm{A}_{1}$. Indeed


FIG. 3. (a) DFT-calculated PDOS of $\mathrm{NO} / \mathrm{Cu}(110)$. Simulated topographies of $\mathrm{NO} / \mathrm{Cu}(110)$ for a constant $\rho=5 \times 10^{-9} e / \AA^{3}$ with (b) $W=-0.2$ and (c) $0.2 \mathrm{eV}$. The positions of the $\mathrm{N}, \mathrm{O}$, and $\mathrm{Cu}$ atoms in the topmost layer are shown by black dots. The image area is $12 \AA \times 12 \AA$. the inelastic image in Fig. 2(d) shows a $A_{1}$-symmetric feature, which suggests that the $\psi_{m, k}$ state is $6 \sigma^{*}$ [Fig. 5(a)]. Compared to the inelastic tunneling components, the additional factor $\delta\left(\varepsilon_{m, \boldsymbol{k}}-\hbar \omega-\varepsilon_{n, \boldsymbol{k}}\right)$ in Eq. (3) imposes a more strict condition to the elastic components because it forces $\psi_{m, \boldsymbol{k}}$ and $\psi_{n, \boldsymbol{k}}$ to be separated in energy by the vibrational quanta $\hbar \omega$. There is no $\psi_{m, k}$ with $\mathrm{A}_{1}$ symmetry fulfilling this condition and, therefore, the elastic contribution is negligible as shown in Fig. 5(b). The IETS map for $\mathrm{FR}_{[1 \overline{1} 0]}$ [Fig. 4(b)], also with $\mathrm{B}_{2}$ symmetry, can be explained with similar arguments. We attribute the slightly weaker IETS intensity of $\mathrm{FR}_{[1 \overline{1} 0]}$ as compared to the $\mathrm{FT}_{[1 \overline{1} 0]}$ one to a possibly smaller electron-vibration coupling $v$. On the other hand, the $\mathrm{FR}_{[001]}$ and $\mathrm{FT}_{[001]}$ modes with $\mathrm{B}_{1}$ symmetry are symmetry-"forbidden" if $2 \pi_{[1 \overline{1} 0]}^{*}$ is the only one contributing to the $\psi_{n, \boldsymbol{k}}$ state. In contrast, $2 \pi_{[001]}^{*}$ with $\mathrm{B}_{1}$ symmetry can yield a nonzero contribution by coupling to $6 \sigma^{*}$ with $\mathrm{A}_{1}$ symmetry. Hence, the nonvanishing IETS signals predicted in our simulations for $\mathrm{FR}_{[001]}$ and $\mathrm{FT}_{[001]}$ [Figs. 4(d) and 4(f)] are in good agreement with the PDOS of Fig. 3(a) that shows a non-negligible presence of the $2 \pi_{[001]}^{*}$ orbital below $E_{\mathrm{F}}$. Actually, we have verified that $\mathrm{FT}(\mathrm{R})_{[001]}$ and not $\mathrm{FT}(\mathrm{R})_{[1 \overline{1} 0]}$ dominate the IETS maps for positive $W$ (not shown).

\section{(a) $\mathrm{v} 196 \mathrm{meV}$ (b) $\mathrm{FR}_{[110]} 42 \mathrm{meV}$ (c) $\mathrm{CM} 35 \mathrm{meV}$}
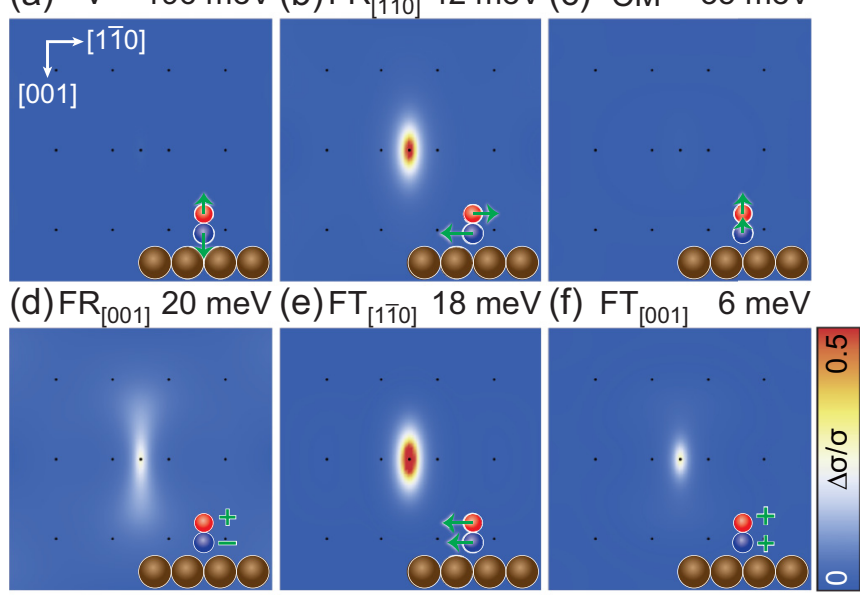

FIG. 4. Simulated IETS maps of NO/Cu(110) with $W=-0.2 \mathrm{eV}$ and $\rho=5 \times 10^{-9} e / \AA^{3}$. Each inset shows a side-view illustration of the corresponding normal mode. The DFT-calculated vibrational quanta $\hbar \omega$ is indicated on top of each panel. The image area is $12 \AA \times 12 \AA$. 


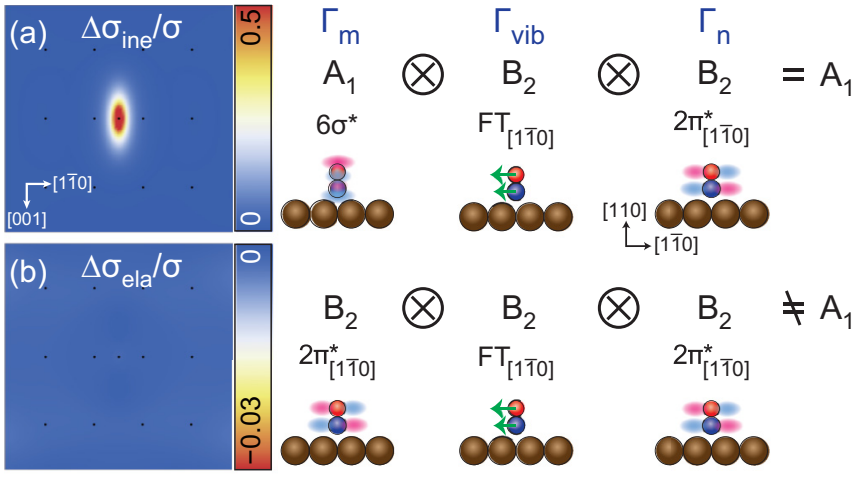

FIG. 5. Calculated intensity maps of (a) inelastic and (b) elastic tunneling components of the $\mathrm{FT}_{[110]}$ mode for $\mathrm{NO} / \mathrm{Cu}(110)$ with $W=$ $-0.2 \mathrm{eV}$ and $\rho=5 \times 10^{-9} e / \AA^{3}$. The possible combination between $\psi_{m, k}$, a vibrational mode, and $\psi_{n, \boldsymbol{k}}$ is shown on the right side of each map. The image area is $12 \AA \times 12 \AA$.

Remarkably, both the experimental and theoretical results discussed so far show that the presence of a resonance at $E_{\mathrm{F}}$ does not necessarily imply a dip in the IETS spectra at $V>0$, as it is the case for $\mathrm{O}_{2}$ on $\operatorname{Ag}(110)$ [15-17,31]. Possible "allowed" combinations for the elastic tunneling of $\mathrm{NO} / \mathrm{Cu}(110)$ correspond to $\Gamma_{n}=\Gamma_{m}=\mathrm{B}_{2}$ and $\Gamma_{\text {vib }}=\mathrm{A}_{1}$, which would lead to a negative IETS map with a dumbbell shape along the [1 $1 \overline{0} 0]$ direction. However, neither the $v$ nor CM modes show any noticeable IETS intensity [Figs. 4(a) and 4(c)], probably because of weak couplings $v$ between the resonance states via these $A_{1}$ modes. This is analogous with IETS for $\mathrm{CO}$ on $\mathrm{Cu}(111)$ with a metal tip, where the $2 \pi^{*}$ orbital of the adsorbate couples predominantly with the FR mode, while coupling between $2 \pi^{*}$ and the $\mathrm{C}-\mathrm{O}$ stretch mode is quite weak $[15,27,28]$.

We next turn to a similar analysis for $\mathrm{NO} / \mathrm{Cu}(001)$. The NO molecule is found to preferentially adsorb upright on the hollow site. An upright configuration bonded to the bridge site is found to be meta-stable (about $170 \mathrm{meV}$ higher in energy). The molecule is practically unpolarized (Table I) as well as $\mathrm{NO} / \mathrm{Cu}(111)$ [48,49]. The calculated PDOS shows that the doubly degenerate $2 \pi_{[110]}^{*}$ and $2 \pi_{[1 \overline{1} 0]}^{*}$ resonances are centered at $E_{\mathrm{F}}$ [Fig. 6(a)], consistent with the experimental STS [Fig. 1(d)]. Simulated topographies of $\mathrm{NO} / \mathrm{Cu}(001)$ successfully reproduce the ring-shaped protrusion [Figs. 6(b) and 6(c)]. Figure 7 shows simulated IETS maps for $\mathrm{NO} / \mathrm{Cu}(001)$ with the schematic illustrations of the normal modes of vibration. The maps of the doubly degenerate FR (38.3 and $38.1 \mathrm{meV})$ and FT (13.7 and $13.5 \mathrm{meV}$ ) modes reveal an intensity spot localized at the center of NO. In the measured IETS [Fig. 2(b)], a single broad peak at $\pm 25 \mathrm{mV}$ is detected. The peak energy seems to coincide with the CM mode $(27 \mathrm{meV})$, but this mode is not expected to be active [Fig. 7(d)]. Therefore the measured broad IETS feature is assigned to the FR and FT modes overlapping each other.

The IETS activity/inactivity of the vibration modes of $\mathrm{NO} / \mathrm{Cu}(001)$ can be explained by "propensity rules" in a similar manner to $\mathrm{NO} / \mathrm{Cu}(110)$. The $\psi_{n, k}$ state is the doubly degenerate $2 \pi^{*}$, indicating that $\Gamma_{n}=\mathrm{E}$. Then there are two possible "allowed" combinations between a vibrational mode
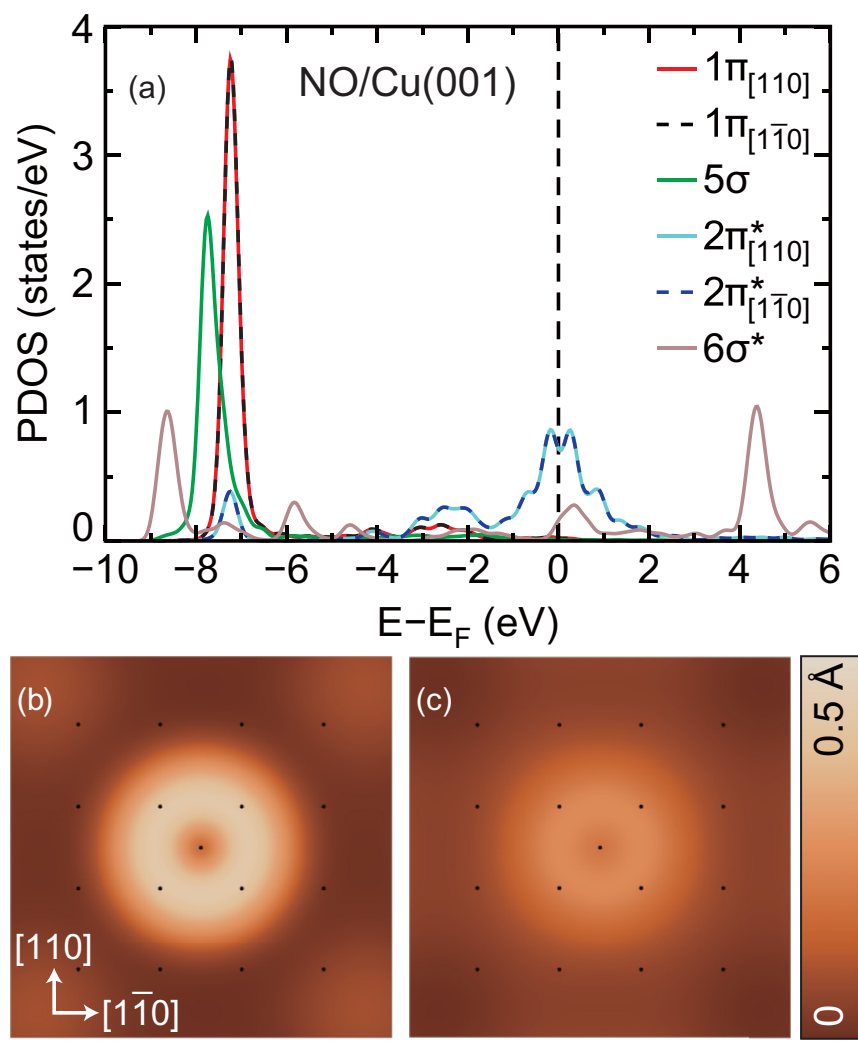

FIG. 6. (a) DFT-calculated PDOS of $\mathrm{NO} / \mathrm{Cu}(001)$. Simulated topographies of $\mathrm{NO} / \mathrm{Cu}(001)$ for $\rho=5 \times 10^{-9} e / \AA^{3}$ with (b) $W=$ -0.2 and (c) $0.1 \mathrm{eV}$. The image area is $12 \AA \times 12 \AA$.

and $\psi_{m, k}$ state: (i) $\Gamma_{\text {vib }}=\mathrm{E}$ and $\Gamma_{m}=\mathrm{A}_{1}$ and (ii) $\Gamma_{\text {vib }}=\mathrm{A}_{1}$ and $\Gamma_{m}=\mathrm{E}$. For the inelastic tunneling, FR and FT modes (E symmetry) is "allowed" if the $\psi_{m, k}$ state is $6 \sigma^{*}\left(\mathrm{~A}_{1}\right.$ symmetry), and then the IETS signal should be localized on top of NO. On the other hand, these modes are "forbidden" for
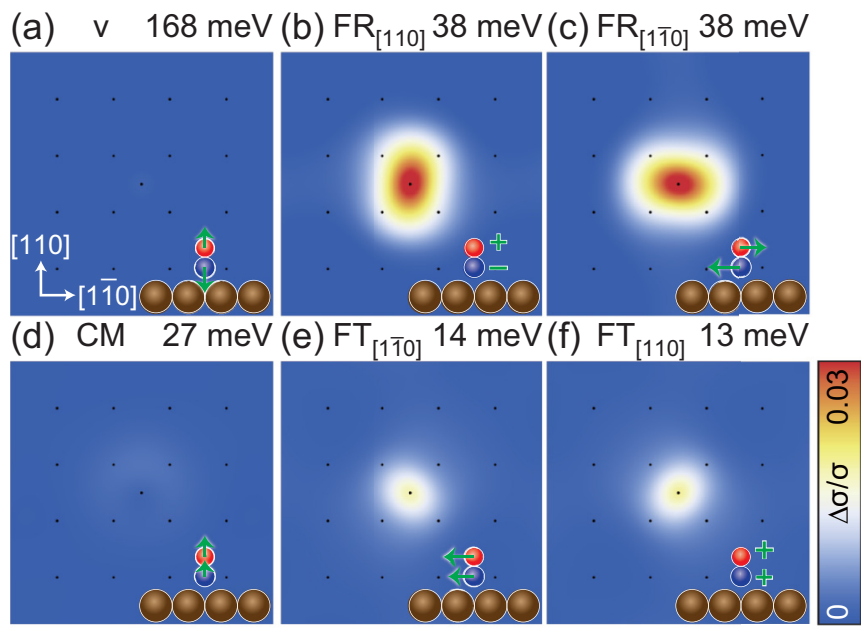

FIG. 7. Simulated IETS maps of NO/Cu(001) with $W=0.1 \mathrm{eV}$ and $\rho=5 \times 10^{-9} e / \AA^{3}$. Each inset shows a side-view illustration of the corresponding normal mode. The DFT-calculated vibrational quanta $\hbar \omega$ is written on top of each panel. The image area is $12 \AA \times$ $12 \AA$. 
the elastic tunneling because combination (ii) is required for on-resonance. These considerations successfully explain the sign and shape of the total IETS maps for the FR and FT modes [Figs. 7(b), 7(c), 7(e), and 7(f)]. The $v$ and CM modes $\left(\mathrm{A}_{1}\right.$ symmetry) correspond to combination (ii) above which would dictate a ring shape (E symmetry) surrounding the molecule in the IETS map. However, we find no noticeable intensity
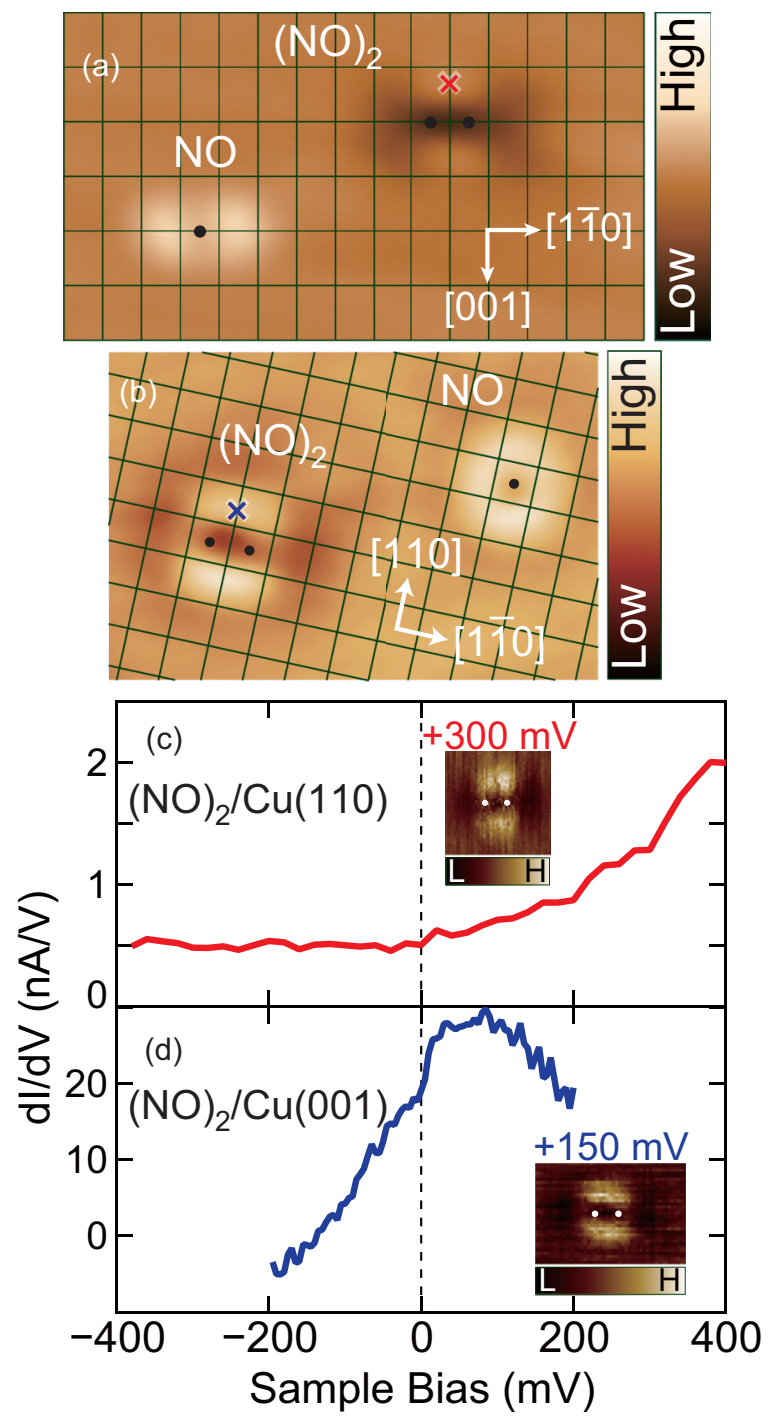

FIG. 8. STM image of a dimer $\left[(\mathrm{NO})_{2}\right]$ together with monomers on (a) $\mathrm{Cu}(110)(V=30 \mathrm{mV}, I=0.5 \mathrm{nA})$ and (b) $\mathrm{Cu}(001)(V=$ $30 \mathrm{mV}, I=5 \mathrm{nA}$ ). The dimer in (a) [(b)] was produced by manipulating two NO molecules in Fig. 1(a) [1(b)] toward each other. $d I / d V$ curves for the dimer on (c) $\mathrm{Cu}(110)$ and (d) $\mathrm{Cu}(001)$. The spectrum in (c) [(d)] was acquired by lock-in detection with the bias modulation of $V_{\text {mod }}^{\text {(rms }}=4$ [1] $\mathrm{mV}$ with the feedback disabled at the tip height to give $30 \mathrm{mV}$ and 0.5 [5] nA over the cross marker in (a) [(b)]. The inset of (c) [(d)] shows a $d I / d V$ spatial map of $(\mathrm{NO})_{2}$ on $\mathrm{Cu}(110)[\mathrm{Cu}(001)]$, which was acquired at $V=300$ [150] $\mathrm{mV}$ with $V_{\mathrm{mod}}^{(\mathrm{rms})}=12 \mathrm{mV}$ with the feedback disabled to give $30 \mathrm{mV}$ and 0.5 [5] nA over the cross in (a) [(b)]. The approximate positions of NO molecules are shown by black dots in (a) and (b) and white dots in the insets of (c) and (d). The image sizes are $38 \AA \times 22 \AA$ for (a), $30 \AA \times 20 \AA$ for (b), $12 \AA \times 12 \AA$ for the inset of (c), and $16 \AA \times$ $11 \AA$ for the inset of (d). in the IETS maps from these modes [Figs. 7(a) and 7(d)], probably resulting from weak couplings $v$ via these modes, as speculated above also for the case of $\mathrm{NO} / \mathrm{Cu}(110)$.

\section{NO dimers on $\mathrm{Cu}(110)$ and $\mathrm{Cu}(001)$}

We also studied on $\mathrm{NO}$ dimers $\left[(\mathrm{NO})_{2}\right]$ on $\mathrm{Cu}(110)$ and $\mathrm{Cu}(001)$. Figures 8(a) and 8(b) show STM images of

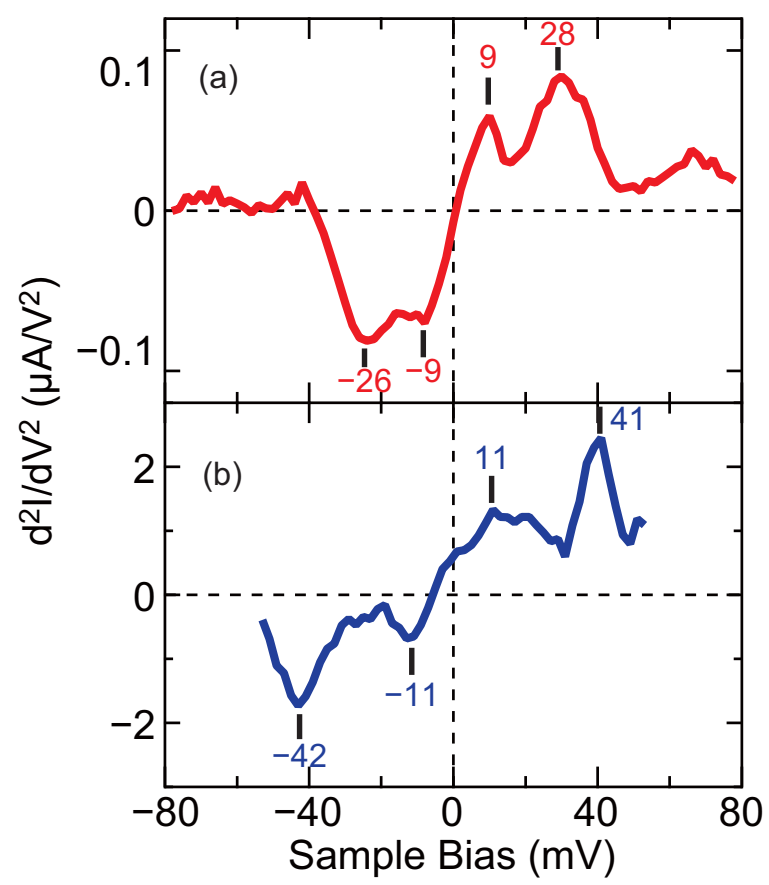

(c) $(\mathrm{NO})_{2} / \mathrm{Cu}(110)$



(d)

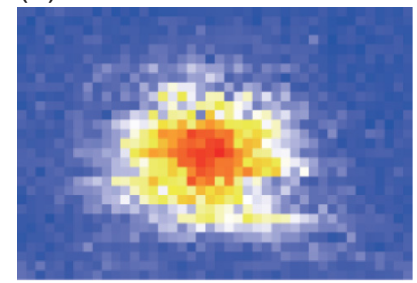

(e) $(\mathrm{NO})_{2} / \mathrm{Cu}(001)$

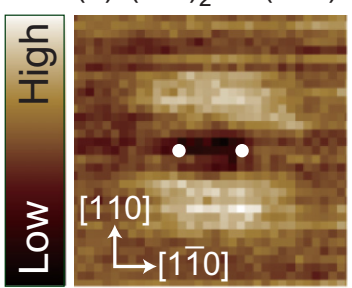

(f) $+40 \mathrm{mV}$

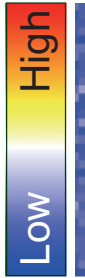



FIG. 9. $d^{2} I / d V^{2}$ curves for the dimer on (a) $\mathrm{Cu}(110)$ and (b) $\mathrm{Cu}(001)$ recorded over on the molecular center (i.e., midpoint between NO and NO). The spectrum in (a) [(b)] was acquired by lock-in detection with the bias modulation of $V_{\mathrm{mod}}^{(\mathrm{rms})}=6[10] \mathrm{mV}$ with the feedback disabled at the tip height to give $30 \mathrm{mV}$ and 0.5 [5] nA. (c) [(e)] Topographic image and (d) [(f)] $d^{2} I / d V^{2}$ spatial map of the dimer on $\mathrm{Cu}(110)[\mathrm{Cu}(001)]$. The topographic image in (c) [(e)] was acquired with $V=30 \mathrm{mV}$ and $I=0.5$ [5] nA to give the tip height for measuring $d^{2} I / d V^{2}$ signal at each point. The map in (d) [(f)] was subsequently acquired at $V=30$ [40] $\mathrm{mV}$ with $V_{\mathrm{mod}}^{(\mathrm{rms})}=12 \mathrm{mV}$ with the feedback disabled. The approximate positions of NO molecules are shown by white dots in (c) and (e). The image sizes are $16 \AA \times$ $11 \AA$ for (c) and (d) and $11 \AA \times 11 \AA$ for (e) and (f). 
$(\mathrm{NO})_{2} / \mathrm{Cu}(110)$ and $(\mathrm{NO})_{2} / \mathrm{Cu}(001)$, respectively, together with a NO monomer. Two NO molecules on each surface $[\mathrm{Cu}(110)$ in Fig. 1(a) and $\mathrm{Cu}(001)$ in Fig. 1(b)] were approached to each other with STM manipulation [35] to yield the dimer. Figure 8(c) [8(d)] shows a $d I / d V$ curve for $(\mathrm{NO})_{2} / \mathrm{Cu}(110)\left[(\mathrm{NO})_{2} / \mathrm{Cu}(001)\right]$ recorded over the cross marker in Fig. 8(a) [8(b)]. The spectrum in Fig. 8(c) shows a shoulder of a peak at $V>200 \mathrm{mV}$, suggesting the resonance state is located around $E_{\mathrm{F}}+0.5 \mathrm{eV}$. The resonance state in Fig. 8(d) is observed at $\sim 50 \mathrm{meV}$ above $E_{\mathrm{F}}$. Insets of Figs. 8(c) and $8(\mathrm{~d})$ show $d I / d V$ spatial maps of $(\mathrm{NO})_{2} / \mathrm{Cu}(110)$ and $(\mathrm{NO})_{2} / \mathrm{Cu}(001)$ recorded at $V=300$ and $150 \mathrm{mV}$, respectively. These intensity maps show an elongated dumbbell shape along a direction perpendicular to the molecular plane. The structure probably reflects the shape of the $2 \mathrm{~b}_{1}$ and/or the $2 \mathrm{a}_{2}$ orbitals (discussed below). The different resonance position between the two substrates originates from the energetic variation between $2 \pi_{[001]}^{*}$ for $\mathrm{NO} / \mathrm{Cu}(110)$ [Fig. 3(a)] and $2 \pi_{[110]}^{*}$ for $\mathrm{NO} / \mathrm{Cu}(001)$ [Fig. 6(a)].


$7 b_{2}$

FIG. 10. (a) DFT-calculated PDOS of $(\mathrm{NO})_{2} / \mathrm{Cu}(110)$. Simulated topographies of $(\mathrm{NO})_{2} / \mathrm{Cu}(110)$ for $\rho=5 \times 10^{-9} e / \AA^{3}$ with (b) $W=$ $-0.1 \mathrm{eV}$ and (c) $W=0.2 \mathrm{eV}$. The image area is $12 \AA \times 12 \AA$. (d) Top-view visualizations of the molecular orbitals computed for the specific $(\mathrm{NO})_{2}$ geometry as obtained on $\mathrm{Cu}(110)$. The absolute value of the isosurfaces is $0.01 \AA^{-3 / 2}$. The positions of $\mathrm{N}$ and $\mathrm{O}$ atoms are shown by red and blue dots, respectively.
$\mathrm{A} d^{2} I / d V^{2}$ curve for $(\mathrm{NO})_{2} / \mathrm{Cu}(110)$ was measured over the center of the dimer [Fig. 9(a)], showing vibrational features at \pm 9 and $\pm 27 \mathrm{mV}$. The latter signal is localized at the center of the dimer, as shown by an IETS spatial map at the corresponding bias [Fig. 9(d)]. An IETS curve and a map for $(\mathrm{NO})_{2} / \mathrm{Cu}(001)$ were also measured in the similar way, revealing vibrational features at \pm 11 and $\pm 41 \mathrm{meV}$ [Fig. 9(b)]. The latter signal is also localized at the center of the dimer [Fig. 9(f)], while the simultaneously-measured topography shows the feature of $2 b_{1}$ and/or $2 a_{2}$ orbitals [Fig. 9(e)].

We also calculated the optimized structures of $(\mathrm{NO})_{2}$ on the $\mathrm{Cu}$ surfaces (see Table I), which are quite similar to those reported in Ref. [46]. We find that the dimers on both surfaces are practically unpolarized. According to the calculated adsorption energies, dimer formation from two monomers on the surfaces is energetically [un-]favorable for $(\mathrm{NO})_{2} / \mathrm{Cu}(110)\left[(\mathrm{NO})_{2} / \mathrm{Cu}(001)\right]$ by the difference $\Delta E_{\text {ads }}=$ $87[-31] \mathrm{meV} / \mathrm{molecule}$. Figures 10 and 11 show calculated PDOS and IETS maps, respectively, for $(\mathrm{NO})_{2} / \mathrm{Cu}(110)$. Similarly, PDOS and IETS maps for $(\mathrm{NO})_{2} / \mathrm{Cu}(001)$ are

(a) $v_{s} 204 \mathrm{meV}$ (b) $\quad v_{a} 195 \mathrm{meV}(\mathrm{c}) \mathrm{FR}_{[110]}^{\mathrm{s}} 60 \mathrm{meV}$


FIG. 11. Simulated IETS maps of $(\mathrm{NO})_{2} / \mathrm{Cu}(110)$ with $W=$ $-0.1 \mathrm{eV}$ and $\rho=5 \times 10^{-9} e / \AA^{3}$. Each inset shows a side-view illustration of the corresponding normal mode. The DFT-calculated vibrational quanta $\hbar \omega$ is written on top of each panel. The image area is $12 \AA \times 12 \AA$. 


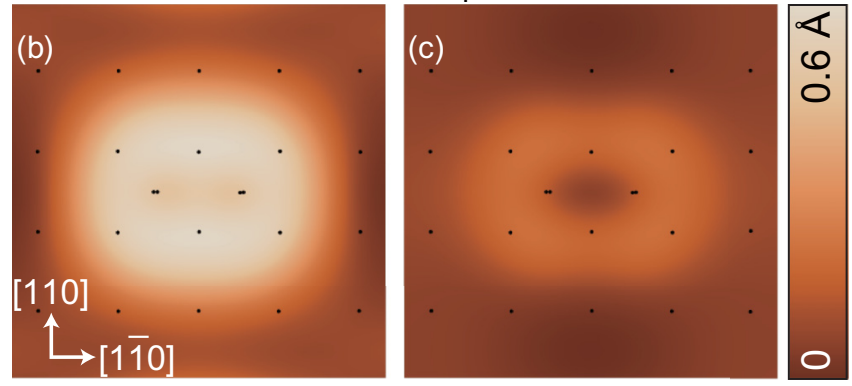

FIG. 12. (a) DFT-calculated PDOS of $(\mathrm{NO})_{2} / \mathrm{Cu}(001)$. Simulated topographies of $(\mathrm{NO})_{2} / \mathrm{Cu}(001)$ for $\rho=5 \times 10^{-9} e / \AA^{3}$ with (b) $W=$ -0.1 and (c) $0.3 \mathrm{eV}$. The image area is $12 \AA \times 12 \AA$.

shown in Figs. 12 and 13. For both systems, according to the calculated PDOS [Figs. 10(a) and 12(a)], the resonance states of $2 b_{1}$ and $2 a_{2}$ orbitals [Fig. 10(d)] are predominantly located near $E_{\mathrm{F}}$, which is compatible with the experimental STS [Fig. 8]. However, the simulated topographies of both dimers [Figs. 10(b), 10(c) for (NO) $)_{2} / \mathrm{Cu}(110)$ and 12(b), 12(c) for $\left.(\mathrm{NO})_{2} / \mathrm{Cu}(001)\right]$ do not reproduce too well the experimental images, mainly because all four hybridized molecular orbitals have tails extending to $E_{\mathrm{F}}$. Nevertheless, the topography of $(\mathrm{NO})_{2} / \mathrm{Cu}(110)\left[(\mathrm{NO})_{2} / \mathrm{Cu}(001)\right]$ with $W=-0.1[0.3] \mathrm{eV}$ resembles more or less the $2 b_{1}$ and/or the $2 a_{2}$ orbitals, and therefore, it is meaningful to compare the simulated IETS for this $W$ with the experimental results. Figures 11 and 13 show the simulated IETS maps for the normal modes for $(\mathrm{NO})_{2} / \mathrm{Cu}(110)$ and $(\mathrm{NO})_{2} / \mathrm{Cu}(001)$, respectively, along with the schematic illustrations of the corresponding vibrations. Since the dimer on both surfaces belong to $C_{2 \mathrm{v}}$ point group, we can consider "propensity rules" [Eq. (4)] for the dimers on the same symmetry conditions. Note that four FT modes of $(\mathrm{NO})_{2} / \mathrm{Cu}(001)$ are degraded from $C_{2 \mathrm{v}}$ due to the (numerically) slightly asymmetric adsorption structure.

First, we find that all of $\mathrm{A}_{1}$ modes, e.g., the symmetric $\mathrm{N}-\mathrm{O}$ stretching mode $v_{\mathrm{s}}$, are (almost) IETS inactive [Figs. 11(a), 11(c), 11(f), 11(i), 13(a), 13(c), and 13(g)]similar to the $A_{1}$ modes of the NO monomers as described above. Next, some of the IETS maps for $B_{1}$ and $A_{2}$ modes have intensities located at the center of the dimer. This can be explained by Eq. (4); the coupling of $\mathrm{B}_{1}\left(\mathrm{~A}_{2}\right)$ modes with the $2 \mathrm{~b}_{1}\left(2 \mathrm{a}_{2}\right)$ resonance state requires $\Gamma_{m}=\mathrm{A}_{1}$. Thus the $\psi_{m, \boldsymbol{k}}$


FIG. 13. Simulated IETS maps of $(\mathrm{NO})_{2} / \mathrm{Cu}(001)$ with $W=$ $0.3 \mathrm{eV}$ and $\rho=5 \times 10^{-9} e / \AA^{3}$. Each inset shows a side-view illustration of the corresponding normal mode. The DFT-calculated vibrational quanta $\hbar \omega$ is written on top of each panel. The image area is $12 \AA \times 12 \AA$.

state probably originates form $7 \mathrm{a}_{1}$ orbital [Fig. 10(d)] and/or $8 \mathrm{a}_{1}$ (bonding $6 \sigma^{*}-6 \sigma^{*}$ ) orbital. Even if the symmetry representations are identical, the intensities are usually different between the normal modes. For example, antisymmetric FR mode along the [001] direction for $(\mathrm{NO})_{2} / \mathrm{Cu}(110)\left[\mathrm{FR}_{[001]}^{\mathrm{a}}\right.$ with $\mathrm{A}_{2}$ symmetry; Fig. 11(g)] has strong intensity whereas $\mathrm{FT}_{[001]}^{\mathrm{a}}\left[\mathrm{A}_{2}\right.$ symmetry; Fig. 11(k)] has no signal. The strength of electron-vibration coupling depends on the vibrational energies and vibrational-displacement distributions [17,50], suggesting that the former mode couples with the resonance state more strongly than the latter.

Finally, the experimental IETS peaks of the dimers can be assigned to the "active" vibrational modes in Figs. 11 and 13 . The higher energy signal for $(\mathrm{NO})_{2} / \mathrm{Cu}(110)(27 \mathrm{meV})$ is assigned to $\mathrm{FR}_{[001]}^{\mathrm{a}}$ and $\mathrm{FR}_{[001]}^{\mathrm{s}}$ modes [Figs. $11(\mathrm{~g})$ and $\left.11(\mathrm{~h})\right]$ with similar energies, whereas the lower one $(9 \mathrm{meV})$ is assigned to $\mathrm{FT}_{[001]}^{\mathrm{s}}$ mode [Fig. 11(1)]. We note that the simulated IETS maps of $\mathrm{FR}_{[001]}^{\mathrm{a} / \mathrm{s}}$ [Figs. 11(g) and 11(h)] appear as an oval protrusion localized between the NO molecules, whereas the corresponding experimental IETS map [Fig. 9(d)] shows an almost round distribution. We assume that the 
experimental distribution is broadened by the measurement with a relatively blunt tip apex [29], and that a burred intensity distribution around the dimer in the simulated maps is also reflected by the experimental map. For $(\mathrm{NO})_{2} / \mathrm{Cu}(001)$, the $41 \mathrm{meV}$ signal is assigned to $\mathrm{FR}_{[110]}^{\mathrm{a}}$ and $\mathrm{FR}_{[110]}^{\mathrm{s}}$ [Figs. 13(e) and 13(f)]. On the other hand, the computed asymmetric maps of the FT modes complicate the assignment of the IETS signal at $11 \mathrm{meV}$. We tentatively assign the peak to $\mathrm{FT}_{4}$ mode [Figs. 13(1)] with reference to the vibrational energy. Comparing Figs. 4 with 11, FT along the [110] direction for the monomer on $\mathrm{Cu}(110)$ is IETS active, whereas for the dimer, $\mathrm{FT}_{[1 \overline{1} 0]}^{\mathrm{s} / \mathrm{a}}$ modes are (almost) inactive while $\mathrm{FT}_{[001]}^{\mathrm{s}}$ mode is active. This variation results from the switching of the electronic states from $B_{2}$ to $B_{1}$ upon the formation of the dimer. Therefore, the IETS measurement for $\mathrm{NO}$ and $(\mathrm{NO})_{2}$ clearly demonstrates a crucial role of symmetry of both electronic and vibrational states involved in the tunneling process.

\section{CONCLUSIONS}

We studied nitric oxide molecules on $\mathrm{Cu}(110)$ and $\mathrm{Cu}(001)$ surfaces by a combination of STM experiments and DFT calculations. We observed isolated NO monomers on $\mathrm{Cu}(001)$ for the first time, and found that they are bonded to the hollow site in an upright configuration. The $2 \pi^{*}$ state of $\mathrm{NO} / \mathrm{Cu}(001)$ remains doubly degenerate and located at the Fermi level. We also fabricated NO dimers on the surfaces by STM manipulation. The resonance states of the dimers are modified due to the covalent bonding between the involved NO molecules. Vibrational fingerprints were experimentally detected in IETS of NO monomers and dimers on $\mathrm{Cu}(110)$ and $\mathrm{Cu}(001)$. The simulated IETS successfully reproduce the experimental threshold energies and spatial distributions, and the active modes are concluded to be the FR and FT modes along the specific $\mathrm{Cu}$ surface directions. On the basis of "propensity rules", the inelastic signals can be explained in a qualitative manner.

\section{ACKNOWLEDGMENTS}

The authors acknowledge H. Ueba for fruitful discussions and thank N. Lorente for providing us his codes to perform the IETS calculations. A.S. acknowledges the support by JSPS KAKENHI Grants No. JP12J00219 and No. JP15H06127. M.A. and T.F. acknowledge financial support by the Basque Departamento de Educación, Universidades e Investigación, the University of the Basque Country UPV/EHU (Grant No. IT-756-13) and by the Spanish Ministerio de Economía y Competitividad (FIS2013-48286-C2-2-P and MAT-201346593-C6-2-P, respectively). T.F. also acknowledges support by the European Union FP7-ICT project PAMS (Contract No. 610446). Computational resources were provided by the DIPC computing center.

\section{APPENDIX: BACKGROUND SUBTRACTION OF $d I / d V$ AND $d^{2} I / d V^{2}$ SPECTRA}

Figure 14(a) shows a series of $d^{2} I / d V^{2}$ spectra measured across a NO monomer on $\mathrm{Cu}(110)$ at intervals of $1.9 \AA$ along the [1필 direction. Spectrum i recorded over the bare $\mathrm{Cu}$ surface [inset of Fig. 14(b)] was used as the background

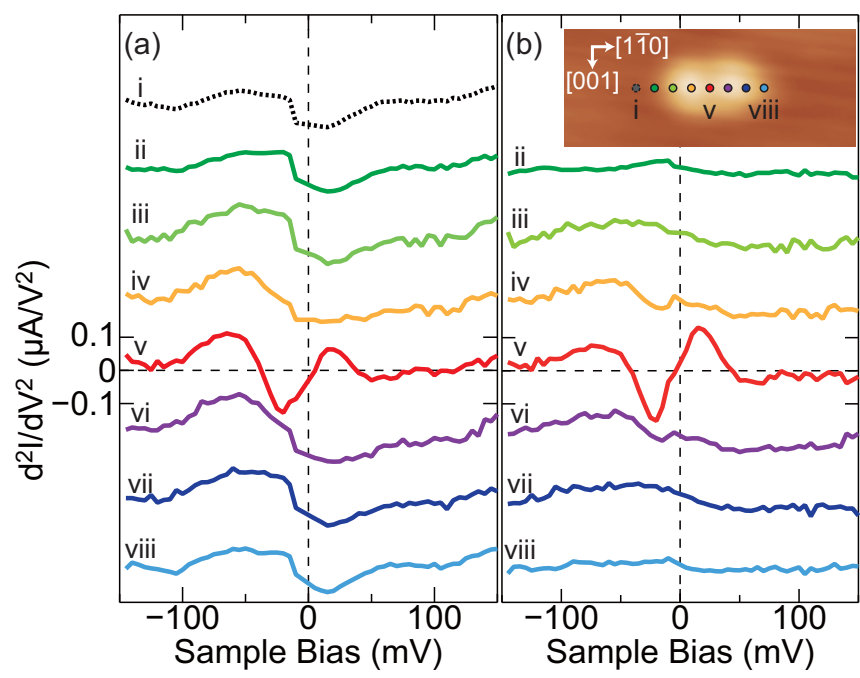

FIG. 14. Spatially resolved $d^{2} I / d V^{2}$ curves of $\mathrm{NO} / \mathrm{Cu}(110)$ (a) before and (b) after background subtraction. Each spectrum was recorded over the same color dot shown in the inset STM image $(V=30 \mathrm{mV}, I=0.5 \mathrm{nA})$. The spectra were acquired by lock-in detection with the bias modulation of $V_{\mathrm{mod}}^{(\mathrm{rms})}=6 \mathrm{mV}$ with the feedback disabled at the tip height to give $30 \mathrm{mV}$ and $0.5 \mathrm{nA}$. The image area for the inset of (b) is $30 \AA \times 12 \AA$.

curve. After background subtraction, as shown in Fig. 14(b), Spectra ii and viii have no feature whereas Spectrum $v$ gives a peak-and-dip feature at $\pm 19 \mathrm{mV}$ [see Fig. 2(a)]. We note that Spectra iii-vii show a smooth dip-and-peak structure at $\sim \pm 50 \mathrm{mV}$, corresponding to the derivative of the $2 \pi^{*}$ resonance [Fig. 1(c)].

Figures 15(a)-15(c) show the unsubtracted $d I / d V$ spectra for Figs. 1 and 8. Red and blue dotted curves represent the background spectra recorded over the bare $\mathrm{Cu}$ surfaces. For example, a spike at $\sim-10 \mathrm{mV}$ in Fig. 15 (a), which is probably due to the electronic structure of the tip, is canceled by
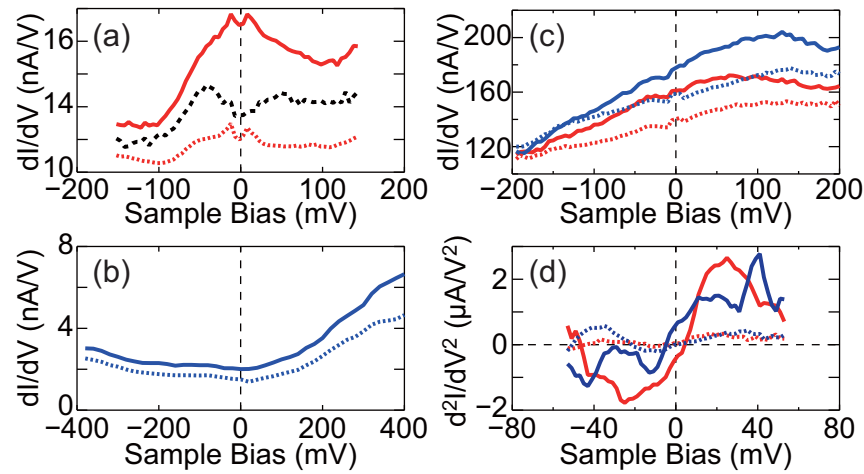

FIG. 15. The unsubtracted $d I / d V$ and $d^{2} I / d V^{2}$ curves. (a) $d I / d V$ spectra of the NO monomer on $\mathrm{Cu}(110)$ recorded over the protrusion (red solid curve) and the molecular center (red solid curve), and the background spectrum recorded over the bare $\mathrm{Cu}$ surface (red dotted curve). (b) $d I / d V$ spectra of the NO dimer on $\mathrm{Cu}(110)$ (solid) and the background spectrum (dotted). (c) $d I / d V$ and (d) $d^{2} I / d V^{2}$ spectra of the NO monomer (red solid) and dimer (blue solid) on $\mathrm{Cu}(001)$, and the background spectra for the monomer (red dotted) and dimer (blue dotted). 
the background subtraction [see Fig. 1(c)]. The background spectra for the NO monomer [red dotted curve in Fig. 15(c)] and dimer (blue dotted) show a similar structure, while the curves recorded over the monomer (red solid) and dimer (blue solid) have different peaks. This indicates that the $2 \pi^{*}(-$ derived) resonance states are located at different energy levels, as shown in Figs. 1(d) and 8(d). Note that the background curves are not identical because of the different tip-sample heights. Figure $15(\mathrm{~d})$ shows the unsubtracted $d^{2} I / d V^{2}$ curves for the $\mathrm{NO}$ monomer (red) and dimer (blue) on $\mathrm{Cu}(001)$, leading to Figs. 2(b) and 9(b), respectively. The background curves (dotted) are featureless as well as on $\mathrm{Cu}(110)$ (Spectra $i$ in Fig. 14), whereas IET signals are detected in the spectra recorded over the molecules (solid).
[1] W. Ho, Single-molecule chemistry, J. Chem. Phys. 117, 11033 (2002).

[2] R. Zhang, Y. Zhang, Z. C. Dong, S. Jiang, C. Zhang, L. G. Chen, L. Zhang, Y. Liao, J. Aizpurua, Y. Luo, J. L. Yang, and J. G. Hou, Chemical mapping of a single molecule by plasmon-enhanced Raman scattering, Nature (London) 498, 82 (2013).

[3] Y. Sugimoto, P. Pou, M. Abe, P. Jelinek, R. Pérez, S. Morita, and $\mathrm{O}$. Custance, Chemical identification of individual surface atoms by atomic force microscopy, Nature (London) 446, 64 (2007).

[4] Y. Kim and H. Song, Investigation of molecular junctions with inelastic electron tunneling spectroscopy, Appl. Spectrosc. Rev. 51, 603 (2016).

[5] R. C. Jaklevic and J. Lambe, Molecular Vibration Spectra by Electron Tunneling, Phys. Rev. Lett. 17, 1139 (1966).

[6] B. C. Stipe, M. A. Rezaei, and W. Ho, Single-molecule vibrational spectroscopy and microscopy, Science 280, 1732 (1998).

[7] B. C. Stipe, M. A. Rezaei, and W. Ho, A variable-temperature scanning tunneling microscope capable of single-molecule vibrational spectroscopy, Rev. Sci. Instrum. 70, 137 (1999).

[8] H. Gawronski, M. Mehlhorn, and K. Morgenstern, Imaging phonon excitation with atomic resolution, Science 319, 930 (2008).

[9] A. J. Heinrich, J. A. Gupta, C. P. Lutz, and D. M. Eigler, Singleatom spin-flip spectroscopy, Science 306, 466 (2004).

[10] N. Tsukahara, K.-i. Noto, M. Ohara, S. Shiraki, N. Takagi, Y. Takata, J. Miyawaki, M. Taguchi, A. Chainani, S. Shin, and M. Kawai, Adsorption-Induced Switching of Magnetic Anisotropy in a Single Iron(II) Phthalocyanine Molecule on an Oxidized $\mathrm{Cu}(110)$ Surface, Phys. Rev. Lett. 102, 167203 (2009).

[11] N. Lorente and M. Persson, Theory of Single Molecule Vibrational Spectroscopy and Microscopy, Phys. Rev. Lett. 85, 2997 (2000).

[12] N. Lorente and M. Persson, Theoretical aspects of tunnelingcurrent-induced bond excitation and breaking at surfaces, Faraday Discuss. 117, 277 (2000).

[13] N. Lorente, M. Persson, L. J. Lauhon, and W. Ho, Symmetry Selection Rules for Vibrationally Inelastic Tunneling, Phys. Rev. Lett. 86, 2593 (2001).

[14] N. Lorente, Mode excitation induced by the scanning tunneling microscope, Appl. Phys. A 78, 799 (2004).

[15] M. Paulsson, T. Frederiksen, H. Ueba, N. Lorente, and M. Brandbyge, Unified Description of Inelastic Propensity Rules for Electron Transport through Nanoscale Junctions, Phys. Rev. Lett. 100, 226604 (2008).

[16] M. Alducin, D. Sánchez-Portal, A. Arnau, and N. Lorente, Mixed-Valency Signature in Vibrational Inelastic Electron
Tunneling Spectroscopy, Phys. Rev. Lett. 104, 136101 (2010).

[17] S. Monturet, M. Alducin, and N. Lorente, Role of molecular electronic structure in inelastic electron tunneling spectroscopy: $\mathrm{O}_{2}$ on Ag(110), Phys. Rev. B 82, 085447 (2010).

[18] S. Paavilainen and M. Persson, Vibrational assignments and line shapes in inelastic tunneling spectroscopy: $\mathrm{H}$ on $\mathrm{Cu}(100)$, Phys. Rev. B 74, 085417 (2006).

[19] N. Okabayashi, M. Paulsson, H. Ueba, Y. Konda, and T. Komeda, Inelastic Tunneling Spectroscopy of Alkanethiol Molecules: High-Resolution Spectroscopy and Theoretical Simulations, Phys. Rev. Lett. 104, 077801 (2010).

[20] S. R. Burema, K. Seufert, W. Auwarter, J. V. Barth, and M.-L. Bocquet, Probing nitrosyl ligation of surface-confined metalloporphyrins by inelastic electron tunneling spectroscopy, ACS Nano 7, 5273 (2013).

[21] L. J. Lauhon and W. Ho, Single-molecule vibrational spectroscopy and microscopy: $\mathrm{CO}$ on $\mathrm{Cu}(001)$ and $\mathrm{Cu}(110)$, Phys. Rev. B 60, R8525 (1999).

[22] A. J. Heinrich, C. P. Lutz, J. A. Gupta, and D. M. Eigler, Molecule cascades, Science 298, 1381 (2002).

[23] K. Makoshi and N. Mingo, Theory of inelastic scanning tunneling spectroscopy, Surf. Sci. 502-503, 34 (2002).

[24] G. Teobaldi, M. Peñalba, A. Arnau, N. Lorente, and W. A. Hofer, Including the probe tip in theoretical models of inelastic scanning tunneling spectroscopy: $\mathrm{CO}$ on $\mathrm{Cu}(100)$, Phys. Rev. B 76, 235407 (2007)

[25] L. Vitali, R. Ohmann, K. Kern, A. Garcia-Lekue, T. Frederiksen, D. Sanchez-Portal, and A. Arnau, Surveying molecular vibrations during the formation of metal-molecule nanocontacts, Nano Lett. 10, 657 (2010).

[26] R. Arafune, H.-J. Shin, J. Jung, E. Minamitani, N. Takagi, Y. Kim, and M. Kawai, Combined scanning tunneling microscopy and high-resolution electron energy loss spectroscopy study on the adsorption state of $\mathrm{CO}$ on $\operatorname{Ag}(001)$, Langmuir 28, 13249 (2012).

[27] A. Garcia-Lekue, D. Sanchez-Portal, A. Arnau, and T. Frederiksen, Simulation of inelastic electron tunneling spectroscopy of single molecules with functionalized tips, Phys. Rev. B 83, 155417 (2011).

[28] E. T. R. Rossen, C. F. J. Flipse, and J. I. Cerdá, Lowest order in inelastic tunneling approximation: Efficient scheme for simulation of inelastic electron tunneling data, Phys. Rev. B 87, 235412 (2013).

[29] N. Okabayashi, A. Gustafsson, A. Peronio, M. Paulsson, T. Arai, and F. J. Giessibl, Influence of atomic tip structure on the intensity of inelastic tunneling spectroscopy data analyzed by combined scanning tunneling spectroscopy, force microscopy, and density functional theory, Phys. Rev. B 93, 165415 (2016). 
[30] Ch. Xu, C. L. Chiang, Z. Han, and W. Ho, Nature of Asymmetry in the Vibrational Line Shape of Single-Molecule Inelastic Electron Tunneling Spectroscopy with the STM, Phys. Rev. Lett. 116, 166101 (2016).

[31] J. R. Hahn, H. J. Lee, and W. Ho, Electronic Resonance and Symmetry in Single-Molecule Inelastic Electron Tunneling, Phys. Rev. Lett. 85, 1914 (2000).

[32] J. Guo, J.-T. Lu, Y. Feng, J. Chen, J. Peng, Z. Lin, X. Meng, Z. Wang, X.-Z. Li, E.-G. Wang, and Y. Jiang, Nuclear quantum effects of hydrogen bonds probed by tipenhanced inelastic electron tunneling, Science 352, 321 (2016).

[33] N. Liu, C. Silien, W. Ho, J. B. Maddox, S. Mukamel, B. Liu, and G. C. Bazan, Chemical imaging of single 4, 7, 12, 15-tetrakis[2.2]paracyclophane by spatially resolved vibrational spectroscopy, J. Chem. Phys. 127, 244711 (2007).

[34] K. J. Franke, G. Schulze, and J. I. Pascual, Excitation of JahnTeller active modes during electron transport through single $\mathrm{C}_{60}$ molecules on metal surfaces, J. Phys. Chem. Lett. 1, 500 (2010).

[35] A. Shiotari, Y. Kitaguchi, H. Okuyama, S. Hatta, and T. Aruga, Imaging Covalent Bonding Between Two NO Molecules on Cu(110), Phys. Rev. Lett. 106, 156104 (2011).

[36] A. Shiotari, S. Hatta, H. Okuyama, and T. Aruga, Role of hydrogen bonding in the catalytic reduction of nitric oxide, Chem. Sci. 5, 922 (2014).

[37] A. Shiotari, S. Hatta, H. Okuyama, and T. Aruga, Formation of unique trimer of nitric oxide on $\mathrm{Cu}(111)$, J. Chem. Phys. 141, 134705 (2014).

[38] J. P. Perdew, K. Burke, and M. Ernzerhof, Generalized Gradient Approximation Made Simple, Phys. Rev. Lett. 77, 3865 (1996).

[39] G. Kresse and J. Furthmüller, Efficient iterative schemes for ab initio total-energy calculations using a plane-wave basis set, Phys. Rev. B 54, 11169 (1996).

[40] P. E. Blöchl, Projector augmented-wave method, Phys. Rev. B 50, 17953 (1994).
[41] G. Kresse and D. Joubert, From ultrasoft pseudopotentials to the projector augmented-wave method, Phys. Rev. B 59, 1758 (1999).

[42] M. Methfessel and A. T. Paxton, High-precision sampling for Brillouin-zone integration in metals, Phys. Rev. B 40, 3616 (1989).

[43] N. Gonzalez-Lakunza, N. Lorente, and A. Arnau, Chemisorption of sulfur and sulfur-based simple molecules on $\mathrm{Au}(111)$, J. Phys. Chem. C 111, 12383 (2007).

[44] C. M. Kim, C.-W. Yi, and D. W. Goodman, Adsorption and reaction of $\mathrm{NO}$ on $\mathrm{Cu}(100)$ : an infrared reflection absorption spectroscopic study at 25 K, J. Phys. Chem. B 106, 7065 (2002).

[45] X.-H. Cui and X.-M. Duan, The stability and electronic properties of Pt-modified $\mathrm{Cu}(110)$ and $\mathrm{Cu}(111)$ in the absence/presence of small molecules: a density-functional theory modeling, J. Phys.: Condens. Matter 28, 085001 (2016).

[46] A. X. Brión-Ríos, D. Sánchez-Portal, and P. Cabrera-Sanfelix, $\mathrm{NO}$ adsorption on $\mathrm{Cu}(110)$ and $\mathrm{O}(2 \times 1) / \mathrm{Cu}(110)$ surfaces from density functional theory calculations, Phys. Chem. Chem. Phys. 18, 9476 (2016).

[47] A. Shiotari, T. Mitsui, H. Okuyama, S. Hatta, T. Aruga, T. Koitaya, and J. Yoshinobu, Configuration change of NO on $\mathrm{Cu}(110)$ as a function of temperature, J. Chem. Phys. 140, 214706 (2014).

[48] M. Gajdoš, J. Hafner, and A. Eichler, Ab initio densityfunctional study of NO on close-packed transition and noble metal surfaces: I. molecular adsorption, J. Phys.: Condens. Matter 18, 13 (2006).

[49] A. A. B. Padama, H. Kishi, R. L. Arevalo, J. L. V. Moreno, H. Kasai, M. Taniguchi, M. Uenishi, H. Tanaka, and Y. Nishihata, NO dissociation on $\mathrm{Cu}(111)$ and $\mathrm{Cu}_{2} \mathrm{O}(111)$ surfaces: A density functional theory based study, J. Phys.: Condens. Matter 24, 175005 (2012).

[50] S. R. Burema, N. Lorente, and M.-L. Bocquet, A theoretical rationalization of a total inelastic electron tunneling spectrum: the comparative cases of formate and benzoate on $\mathrm{Cu}(111)$, J. Chem. Phys. 136, 244507 (2012). 